\title{
AIDS now a tractable disease?
}

Hopes that drug therapy will improve the lot of those infected with HIV have brightened, but it is too soon to tell what the consequences will be for the general spread of the disease.

AIDS has come to stay. So much is vividly illustrated by the inexorable increase of the numbers of those infected with HIV (human immunodeficiency virus), especially in the United States. When more than one per cent of the sexually adult population carries the infecting virus, and when there is no prospect of a downturn, it seems plain that the disease may well become as important a source of mortality among those in middle life as, for example, tuberculosis was in the 1930s. But there is also some good news. The spread of AIDS infection by heterosexual sexual intercourse appears not to have been as rapid as some feared just two or three years ago; at least in the short run, that is something to be grateful for. And now, there is the prospect that drug therapy may at least abate the development of overt AIDS in infected people. That many individuals may be helped to live longer, perhaps even to avoid the development of overt AIDS, is naturally welcome, but it is even more important that drug therapy could serve as a firebreak in the spread of infection, eventually limiting the social damage AIDS will cause.

But optimism should be constrained. Compared with the magnitude of the social problems already occasioned in places such as the United States by the spread of AIDS, the new developments (see Nature 340, 581; 24 August 1989) are but straws at which to clutch. The drug called AZT (otherwise the Wellcome drug known as 'Retrovir'), hitherto used in the palliation of overt AIDS, has been approved by the US Public Health Service as a prophylatic for use among those infected with the virus HIV. Experience so far seems to show that the onset of overt AIDS can be delayed, in about half those treated, for at least a couple of years (the duration of the trial). Then approval has also (and commendably quickly) been given for the preliminary testing in human beings of Genentech's clever synthetic protein, called immunoadhesin, made from molecules which combine the receptor molecule CD4 and part of the immunoglobin molecule IgG1: but the first step is to tell whether the drug can be safely used in people: clinical trials proper will begin only in 1990 .

Only the first of these developments can influence the immediate course of the spread of AIDS, and it is too soon to tell what the effects will be. For one thing, the use of AZT will be limited by the scarcity of the drug, based as it is on a natural product derived from herring sperm. Another way of putting that is to say that not many people infected by HIV and excluded from health insurance schemes (often because of their infection) will be able to afford the annual cost of prophylaxis (in excess of $\$ 6,000$ ) out of their own pockets. Moreover, the wider benefits of prophylaxis remain to be determined. When so little is known of the infectiveness of people carrying HIV at the various stages between first infection and the development of overt AIDS, it is too soon to know the extent to which general use of AZT among carriers of HIV will reduce the spread of infection. (The bizarre possibility that, by prolonging the period of normalcy of the infected, general use of AZT might increase the potential for spreading infection cannot be dismissed, but seems unlikely.) On general grounds, if eventually shown to be effective, Genentech's immunoadhesin (which would remove circulating virus from the bloodstream) promises to have a more direct influence on the infection rate, but only time will tell.

The practical question remains of how these potential benefits may be secured not merely for those infected, but for society at large (which has a vital interest in any impediment to the spread of infection). The difficulties should not be underestimated. The physical scarcity of AZT is something to be reckoned with, and at the beginning will almost certainly limit the use of the drug to those carriers of HIV whose T-cells are already depleted (which is what the US Public Health Service recommends), but that will not extinguish demand from infected people (and their physicians) in whom there are no pathological signs. The cost of this development, especially in the United States, will be considerable.

Even if most of those infected with HIV are covered by health insurance schemes, so that the whole cost does not come from their pockets, their treatment will not be free, but will be reflected in the insurance premiums that everybody pays. At present prices, the aggregate cost might exceed $\$ 1,000$ million a year, by which yardstick Senator Edward Kennedy's proposal that there should be an extra $\$ 30$ million in the US budget to cover the cost of treating the uninsured seems over-modest. Yet this is an area of public expenditure in which, the US budget deficit notwithstanding, even the possibility that the wider use of AZT will be a firebreak in the spread of infection should be seized with energy - and at whatever cost is necessary. The emergence of drug prophylaxis, however insubstantial it may be at present, is one of the few cheerful developments in the past five years. 